\title{
LA SEGMENTACIÓN DEL MERCADO DE TRABAJO *
}

(Estudio empírico sobre el colectivo de ingenieros industriales en Cataluña)

\author{
José Luis Crespán Echegoyen \\ (Universitat Autònoma de Barcelona)
}

\section{A MODO DE PREAMBULO}

La crisis económica, que afecta de manera generalizada a todos los ámbitos de lo social y muy particularmente al sistema productivo, ha lanzado a un primer plano de reflexión y de discusión una serie de cuestiones referidas al sistema de relaciones laborales y particularmente las concernientes al mercado de trabajo. Es normal que ocurra así cuando las cifras de parados en el país sobrepasan los seis dígitos, cuando la inmensa mayoría de los jóvenes no encuentran un puesto de trabajo al finalizar su período formativo y cuando el descmpleo empieza a ser notable entre titulados universitarios.

En nuestro caso el abordaje dei tema del mercado de trabajo es anterior y basado en el desarrollo de una actividad investigadora, tanto en el Instituto de Ciencias de la Educación de la Universidad Autónoma de Barcelona como en el Colegio Oficial de Ingenieros Industriales de Cataluña, que, naturalmente, no se centraba en las cuestiones derivadas de la crisis económica sino más bien en las cuestiones que se reconocen bajo el epí. grafe de «sociología de las profesiones».

* Este artícuio es un resumen de la tesis doctoral La segmentación del mercado de trabajo de los ingenieros industriales en Cataluña, Universidad Autónoma de Bazcelona, 1982. 
Sintéticamente expresados éstos son los elementos sobre los que se articula el contexto teórico y crítico en el que se desenvuelve la presente investigación sobre el mercado de trabajo de los ingenieros industriales en Cataluña. Pero como toda investigación, ésta surgió también de una insatísfacción. Cuando en el gabinete de estudios del COIIC empezamos a analizar las encuestas de salarios y atendíamos a las consultas de los profesionales, lógicamente preocupados por el tema de las retribuciones, empezó a fragusar una inquietud enraizada en lo que por aquel entonces llamábamos incoherencias o indeterminaciones del metcado de trabajo.

El mercado de trabajo presentaba unas opacidades a la lectura directa que no permitían ser interpretadas a la luz de la teoría consolidada. En aquellos momentos en el seno del Gabinete se estaba trabajando sobre el proceso de salarización de los técnicos y profesionales, trabajo que pretendía fundamentalmente contestar a la imagen tradicional que continuaba siendo dominante a nivel del colectivo, y que tomaba como punto de arranque el cambio estructural en el sistema productivo $y$, en consecuencia, el cambio de papel de los técnicos y profesionales en el interior de dicho sistema.

La constatación de las indeterminaciones existentes en el metcado de trabajo y la toma de conciencia de la complejidad del mismo, para las que la teoria resultaba insuficiente, nos condujeron a la necesidad de realizar un replanteamiento radical del propio concepto de metcado de trabajo y de la metodología para analizarlo. El mercado de trabajo resultaba un concepto teórico laxo que se deshacía en cuanto se intentaba buscar su estructutación por la vía de detección de regulatidades que fueran un poco más allá del sentido común, y por otra parte la metodología basada en los estrictos análisis de la causalidad se mostraba incapaz de arrojar luz nueva sobre los puntos oscuros del tema. Resultaba imprescindible desbloquear una y otra, teoría y metodología, para poder progresar en la comprensión del fenómeno.

Es precisamente en esta perspectiva en la que iniciamos los trabajos que han conducido al presente resultado. De una parte mediante la búsqueda de referentes teóricos que, desde abordajes diferentes, discutieran los presupuestos tradicionales y esbozaran nuevas interptetaciones del mercado de trabajo, y de otra poniendo a punto un diseño metodológico que resultara pertinente con respecto a estas nuevas interpretaciones y que permitiera contestar concluyentemente a determinadas conceptualizaciones sobre el mercado de trabajo. 
La segmentación del mercado de trabajo

\section{ALGUNAS CONSIDERACIONES \\ SOBRE EL MERCADO DE TRABAJO}

Por lo que respecta al desarrollo teórico de la investigación se trataba de revisar los presupuestos básicos que han caracterizado a la conceptualización neoclásica del mercado de trabajo, en primet lugar, para posteriormente explorar todas aquellas aportaciones que de manera parcial o global pusieran en cuestión dichos presupuestos básicos.

La conceptualización neoclásica del mercado de trabajo venía caracterizada pot el hecho de entender este mercado como un espacio homogéneo y neutro, regido por mecanismos de ajuste estrictamente internos y en el que los agentes intervinientes —oferta y demanda- tienden estructuralmente al equilibtio y son igualmente importantes. Ello supone, además, que los salarios se forman automáticamente al operar la oferta y la demanda en un espacio que se entiende como un espacio puramente algebraico en el que no interfieren elementos externos como por ejemplo el propio desarrollo del proceso productivo.

Esta conceptualización encontraba complemento teórico en los presupuestos fundamentales de la ueconomía de la educación» y en la «teoría del capital humano» para las que, expresado sintéticamente, la educación es un sinónimo de desarrollo económico y social, la productividad viene básicamente determinada por los niveles educativos de los trabajadores y los salarios responden a dicha productividad.

$\mathrm{La}$ exploración sistemática de las aportaciones más recientes a! tema nos permitió detectat toda una gama de elaboraciones, tanto en el sentido de la reformulación de la teoría neoclásica en las que se pretendía dar explicación de alguna de las disfunciones observadas por la vía de la restricción del modelo, como en el del cuestionamiento implícito o explícito del mismo y que puede afectar a alguna o a todas las características antes mencionadas.

$D::$ in inceterminaciones que aparecían en las primeras, que se ven obligadas muchas veces a dar entrada a elementos externos a los mecanismos de ajuste propios del mercado, y de las matizaciones y formalizaciones de las segundas, concluimos una conceptualización del metcado de trabajo antagónicamente definida respecto a la neoclásica.

Los estudios de Arrow y Capron (1959) sobre la escasez dinámica de la oferta y los de Blaug, Layard y Woodhall (1969) sobre el excedente de la misma, nos permitieron constatar la necesidad de incorporar elementos externos al modelo. Los de Berg (1970) por un lado y los de Rawlins y Ullman (1974) por otro, permitieron establecer la no concordancia entre niveles de remuneración y niveles de productividad a partir del análisis del 
fenómeno del credentialism. Los de Gintis y Bowles (1976) ponían en cuestión el papel de los niveles educativos, y en general del sistema educativo, en lo que se refiere a la estructuración del metcado. Los de Piore (1973), Doeringer (1971) y Thurow (1975) demostraban la existencia de segmentos diferenciados en el interior del mercado; etcétera. Todo ello nos llevó a una conceptualización del mercado de trabajo como un sistema plural y con lógicas diversificadas en el que la demanda desarrolla un papel hegemónico mediante actuaciones basadas no siempre en criterios económicos; en el que la formación de los salarios resulta de una confrontación entre los agentes intervinientes; y en cl que los salarios vienen determinados por el coste del factor trabajo en el proceso productivo. Por otra parte el grado de desarrolio de este proceso es determinante a la hora de configurar situaciones diversas de mercado.

En definitiva, esta exploración nos permitió rechazar los supuestos teóricos básicos de la ueconomía de la educación» y de la «teoría del capital humano" y articular una estructuración del mercado de trabajo coherente con la conceptualización del mismo artiba explicitada. En este sentido fue muy útil la revisión de los diversos estudios en los que se plantea la cuestión de la división, estratificación o segmentación del mercado de trabajo así como la discusión de los criterios utilizados.

Así, se revisaron las tesis de la «balcanización», las interpretaciones de Paci (1973), las teorías de Bowles (1976) y Gintis (1976), las tesis de Gordon (1972), de Doeringer (1971) y de Piore (1973), las de Vietorisz y Harrison (1973), Cotiat (1978) y las de Thurow (1975), como elementos más importantes de este análisis de la fragmentación. Naturalmente no todas ellas son concurrentes en el número de fragmentos significativos ni en los criterios de fragmentación, pero en la práctica totalidad de ellos subyace la hipótesis de discontinuidad entre los diversos segmentos que nosotros asumimos.

En particular, en la investigación se ha adoptado la línea de análisis de Thutow (1975), para quien el mercado de trabajo se estructura como una cola de colas en la que los diversos criterios realizan una primera ordenación de los candidatos en grupos relativamente homogéneos, y sobre la que inciden después ordenaciones particulates generalmente detivadas de los criterios de los empleadores. 
Realizado este trabajo teótico resultaba necesario aplicar la restricción al objeto específico de nuestra investigación, el mercado de trabajo de los ingenietos industriales. Se hacia preciso matizar alguna de las cuestiones planteadas anteriormente por to que se refere al cambio experimentado en el papel de los técnicos en el proceso productivo, haciendo especial insis. tencia en las tres dimensiones básicas sobre las que discurre la dinámica de este mercado de trabajo específico. Dichas dimensiones son: 1) la generación en el interior del sistema productivo de funciones eminentemente técnicas, 2) el incremento considerable de la proporción de técnicos en la estructura de las empresas y 3 ) el progresivo distanciamiento del grueso de los técnicos de las funciones gerenciales. Sobre esta base es sobre la que se genera la diversificación de situaciones de mercado para los ingenieros, tompiendo la hipótesis de continuidad de dicho mercado con la que tradicionalmente se había operado.

Las diferentes condiciones técnicas y organizativas bajo las que se generan los nuevos puestos de trabajo van configurando lógicas distintas de estructuración para los contingentes de profesionales que se van incorporando al mercado de trabajo.

Puestas así las cosas, los objetivos de nuestra investigación son, en primer lugar, la demostración de la no existencia de una estructura lineal del mercado de trabajo de los ingenieros industriales o, lo que es lo mismo, la comprobación de que se da una estructura multisegmentada del mercado de trabajo y, en segundo lugar, la búsqueda de los criterios y lógicas que rigen esta segmentación.

La consecución de estos objetivos ha exigido el diseño de una metodología ex novo, sin, precedentes a los que tomar como referencia, y basada en las técnicas del análisis de datos con poca tradición en nuestro país. Por otra parte cabe señalar como rasgo característico de este diseño metodoló. gico su voluntario decantamiento hacia lo que puede ser explicitado como Ia maximización de la potencialidad heurística e interpretativa.

Por lo que se tefiere al primer objetivo, hemos utilizado un procedimiento recurrente que tiende, primero, a constatar la existencia de diferencias significativas en las remuneraciones de los ingenieros y a determinar la capacidad discriminatoria, con respecto a los salarios, de las variables estructurales que definen al individuo, al puesto de trabajo y a la empresa en que lo desempeña y, posteriormente, a contrastar la ordenación inducida por los salarios percibidos con la clasificación de los profesionales realizada en base a las mencionadas variables estructurales. Si ciertamente se produ- 
cen diferencias significativas entre los salarios y resulta negativa la contrastación de ambas otdenaciones, estaremos en condiciones de afirmar la no existencia de una estructuta lineal del mercado de trabajo.

Pata la comprobación de las diferencias salariales se ha utilizado el análisis de la varianza como instrumental metodológico más adecuado, ya que lo que interesa es la comparación de las medias de remunetación entre los diferentes grupos de ingenieros y el establecimiento de la significación de las mismas mediante la comparación entre la varianza entre grupos y la varianza intragrupos. Se trata de una metodología convencional que no precisa de mayores explicaciones.

La metodología utilizada para realizar el contraste es algo más compleja ya que en ella se combinan diferentes técnicas, en concreto una de las formas del análisis factorial y el análisis discriminante. Las escasas aplicaciones de estas técnicas en investigaciones aplicadas en este país obligan a plantear mínimamente en qué consisten dichas técnicas y a abundar en la justificación de su utilización en el presente trabajo.

El análisis factorial no es un concepto unitario ya que bajo este epígrafe se reconocen diversas técnicas pertinentes a diferentes cuestiones analíticas. La característica común más importante es la capacidad de reducir los datos a una serie de factores o componentes que permiten, de una parte, manipular más eficazmente las grandes bases de datos y, de otra, «visualizar» la estructura de los mismos.

En esta perspectiva el análisis factorial es especialmente indicado para usos exploratorios que permitan la detección de nuevos conceptos estructurantes de la variabilidad de los datos observados y para usos confirmatorios cuando se haya podido establecer con anterioridad alguna hipótesis acerca de la estructuración de los individuos en base a las variables que los califican, y también como tecnología instrumental que permite construir nuevos índices que pueden ser usados como nuevas variables en análisis posteriores.

Sintéticamente podemos decir que el análisis factorial surge a partir de una generalización del análisis de tegresión y tiene como objetivo central la configuración de unos denominados factores que resultan de la combinación lineal de las variables y que son los que mejor explican la variabilidad de la nube de puntos que constituyen los datos observados.

Una de las modalidades del análisis factorial, especialmente diseñada para aquellas situaciones en las que no es posible o no se desea asumir ninguna hipótesis acerca de la estructura general de las variables, es la denominada análisis en componentes principales. Éste, en lugar de suponer que los datos se comportan según un modelo preestablecido, permite a 
través de los factores extraídos reconocer el modelo y calcular los paráme. tros más probables de caracterización del colectivo.

Es esta la modalidad que más nos interesa y la que vamos a utilizar en nuestro análisis. La pertinencia de la misma radica en el becho de que, al no exigir un esquema previo de causalidad entre las variables, se plantea como un modelo abierto en el que la estructuración de los datos no viene condicionada por hipótesis previas y de esta forma se articula en base al carácter probabilístico de las distribuciones de los individuos. En concreto, para nuestra investigación se trata de realizar un análisis en componentes principales que permita calcular las coordenadas de cada individuo sobre los factores resultantes para, a partir de estas coordenadas, establecer una ordenación de los individuos en clases to máximo de homogéneas en cuanto a su composición interna y lo máximo de diferenciadas entre ellias siguiendo un procedimiento topológico de clasificación no jerárquica.

Para hacer esta clasificación de los individuos se ha utilizado un procedimiento diseñado por Lebart, Morineau y Tabard (1977) basado en una aplicación particular del método de las nubes dinámicas y especialmente pensado para ser utilizado con las coordenadas resultantes de un análisis factorial en componentes principales. Tiene como característica principal que la elección de los centros de las nubes se realiza por un procedimiento aleatorio, lo que garantiza el carácter no jerárquico de la clasificación, y que se busca la estabilidad de la misma mediante un proceso de interación que, para conseguir que la clasificación resulte estable, tiene que ser convergente.

Una vez realizada la clasificación de los individuos sin que se haya introducido ningún sesgo al primar determinadas variables, se está en condiciones de contrastarla con la ordenación que inducen los salarios en el mercado de trabajo.

Previamente y como prueba de consistencia de la clasificación establecida se efectúa un análisis discriminante en el que la preordenación es la resultante del proceso de clasificación y en la que las variables discriminatorias son las mismas que han sido utilizadas en el análisis en componentes principales. Se establece la consistencia de la clasificación a partir de la constatación de los elevados porcentajes de individuos que resultan «bien» clasificados, es decir, que coinciden en su pertenencia al mismo grupo según ambos tipos de clasificación. A partir de aquí ya estaremos en condiciones de realizar el contraste antes mencionado.

Para ello se utilizará el mismo test que el arriba descrito, pero utilizando como variable discriminatoria el salario. La interpretación de este segundo análisis discriminante será que si hay un porcentaje elevado de individuos «bien» clasificados para los que las ordenaciones son coincidentes, 
podrá mantenerse la hipótesis de que la estructuración inducida por las variables definidoras de la situación profesional y del individuo y la estructuración inducida por los salatios son paralelas y por lo tanto nos encon. traremos ante una estructura del mercado de trabajo lineal, en la que el salario puede ser entendido como la resultante de una combinación lineal de los factores o variables estructurantes. Si, por el contrario, el porcentaje de individuos «bien» clasificados es bajo, habremos comprobado que ambas ordenaciones no son coincidentes y tendremos que rechazar la hipótesis de una estructura lineal en el mercado de trabajo, teniendo que aceptar la existencia de una estructura compleja del mismo en la que coexisten segmentos cuya lógica de retribución es diferente. Esta segunda posibilidad es la que permite, mediante una demostración a contrario, establecer la corrección de nuestra hipótesis de la existencia de un mercado de trabajo segmentado y refutar los principios básicos de la teoría del capital humano en cuanto a la formación de los salarios y en cuanto a la estructuración del mercado de trabajo.

Por lo que se refiere a la segunda parte de los objetivos propuestos, la búsqueda de criterios y lógicas que rigen esta segmentación, y que obviamente tiene un carácter explotatotio, se ha diseñado una metodología de corte eminentemente heurístico. Una vez comprobada la existencia de una segmentación y sin que por el momento se esté en condiciones de establecer las características de esta segmentación, se plantea la posibilidad de comprobar si se dan estructuraciones diferentes significativamente entre grupos de profesionales diferenciados por su posicionamiento en la escala salarial. Para ello se han construido unos rangos de salarios que distribuyen a la población estudiada en cinco grupos de similar entidad numérica y sobre cada uno de estos grupos se aplica un análisis de componentes principales con el objetivo de ver si realmente hay diferencias notables entre ellos por lo que se tefiere a los factores que mejor explican su variabilidad interna. Dependiendo del grado de difetenciación observado entre las estructuras factoriales, podremos establecer la pertinencia de la división en cinco segmentos.

\section{LA BASE INFORMATIVA UTILIZADA}

La base de datos utilizada en nuestra investigación resulta de la agregación de las encuestas de salatios realizadas por el Colegio Oficial de Ingenieros Industriales de Cataluña, a través de su Gabinete de Estudios, en los años 1976,1977, 1978, 1979 y 1980, que contemplan Ias remunera- 
ciones percibidas por los ingenietos en los años inmediatamente anteriores a cada una de las encuestas.

Si el estudio hubiera tenido como objetivo central la evolución de los salarios, hubiera sido preciso transformar las informaciones salatiales a pesetas constantes; pero en la medida en que lo que se buscaba era la ordenación relativa y estructuración del mercado, esta transformación podía haber supuesto algún sesgo $\sin$ que como contrapartida se viera incrementado su potencial explicativo.

Por lo que se refiere a modificaciones de la base original, no se han hecho más que las necesarias para homogeneizar la información recogida en los sucesivos años, obviando las modificaciones introducidas en el protocolo de tecogida, en la tendencia a mantener la máxima información po. sible para cada una de las variables utilizadas. Sólo se ha optado por recodificaciones con pérdida de información en aquellos casos en que la tecons. trucción resultaba imposible.

Conviene destacar que el presente trabajo utiliza una base informativa no recogida de una forma ad boc para su realización. No obstante, la calidad de la misma es más que suficiente.

Las vatiables utilizadas se configutan en tres grupos de acuerdo con la información que recogen. En primer lugar tenemos las que informan acerca del tipo de empresa en la que trabaja el ingeniero y son: sector de actividad económica de la empresa, tanaño de la misma en cuanto al número de trabajadores que emplea y procedencia del capital. En segundo lugar las que caracterizan propiamente al puesto de trabajo y que, básicamente, recogen información en torno a las funciones desempeñadas y al posicionamiento jerárquico del puesto en el organigrama de la empresa. En este segundo grupo distinguimos: el átea de funciones, el tipo de función de. sempeñado y la distancia en escalones jerárquicos a la getencia.

En tercer lugar tenemos las variables que caracterizan al individuo y que se reducen a la experiencia profesional y a la antiguiedad en el puesto de trabajo. Este conjunto de ocho variables junto al dato de la remuneración percibida es el que ha sido utilizado en todos los análisis del presente trabajo.

Por lo que se refiere al número de individuos contemplados en cada una de las encuestas nos remitimos a la tabla 1. 
«Papers»: Revista de Sociologia

TABLA 1

Total de Ingenieros Industriales que tespondieton a la encuesta de salatios pata los años sucesivos

\begin{tabular}{ccc} 
Año encuesta & $\begin{array}{c}\text { Información } \\
\text { salario año }\end{array}$ & $\begin{array}{c}\text { N. }{ }^{\circ} \text { respuestas } \\
\text { válidas }\end{array}$ \\
\hline 1976 & 1975 & 2.069 \\
1977 & 1976 & 2.287 \\
1978 & 1977 & 2.161 \\
1979 & 1978 & 2.243 \\
1980 & 1979 & 2.108 \\
\hline
\end{tabular}

No se reproducen aquí los análisis de representatividad de estas mues. tras respecto a la población de ingenieros industriales en Cataluna, puesto que este extremo ya viene detallado en las propias publicaciones del COIIC.

Conviene recordar únicamente la estabilidad de las distribuciones de los encuestados respecto de las variables analizadas para los cinco años que constituyen la base de datos del presente trabajo. La conclusión es que la población se encuentra similatmente estructurada para los cinco años y que las modificaciones que pueden observarse responden a modificaciones del mercado de trabajo y no a desiguales conformaciones de las muestras sucesivas. Así, podemos entender las cinco encuestas como lecturas sucesivas realizadas sobre una misma población, lo que nos permitirá detectar las regularidades $\mathrm{y}$ las tendencias de cambio en las condiciones de trabajo de este colectivo y, en definitiva, detectar la estructura del mercado de trabajo en el que se encuentra.

\section{LA VARIABILIDAD SIGNIFICATIVA DE LOS SALARIOS}

Tal y como se apuntó con anterioridad, la variabilidad de los salarios se analiza mediante el análisis de la varianza de las medias salariales grupales definidas por las diversas modalidades de las variables utilizadas en el estudio. Del análisis realizado se desprende una consideración sustantiva para nuestra investigación como es la que afirma que existen diferencias significativas en la temuneración de los ingenietos industriales y, por lo 
tanto, la presunción de que se dan situaciones diferenciadas $(o$, dicho en términos de nuestra investigación, segmentos diferenciados en el mercado de trabajo) se ve cortoborada.

Una segunda consideración es la de que no todas las variables introducidas como elementos de explicación de la vatiabilidad de los salarios cumplen este papel. Se aprecia claramente que mientras las variables que califican al individuo y las que califican al puesto de trabajo tienen un potencial discriminatorio importante, las variables de caracterización estructural de la empresa carecen de él. Ello no quiere decir que las características de la empresa no sean, o puedan ser, elementos importantes en la configuración de los segmentos del mercado de trabajo, sino que en concreto estas variables, que son las que tradicionalmente se han utilizado como caracterizadoras de las situaciones diferentes del mercado, no tienen esa importancia que tópicamente se les ha concedido. De todas formas hay que tener en cuenta que en esta fase han sido consideradas individualmente y a su carácter individual nos tefetimos cuando hablamos de su incapacidad explicativa o discriminante. En las fases posteriores de este trabajo, en que se hacen intervenir todas las variables simultáneamente, es posible que ad. quietan en combinación entre sí o con otras una importancia relativamente mayor.

- Nos permitimos en este resumen no abundar en datos concretos acerca de este análisis de varianza, para los que remitimos al volumen de la tesis. Ello artanca del convencimiento de que no tiene sentido aislar alguno de los datos resultantes, sino que, por el contrario, lo que es ilustrativo es el conjunto de los resultados.

Finalmente hemos de recalcar una cuestión importante. El objetivo de este análisis no es estudiar y valorar el comportamiento y la dinámica de las medias salariales para los diferentes grupos de profesionales, y en este sentido no aparecen comentarios sobre los valores concretos de dichas medias salariales, sino el de buscar los posicionamientos relativos de dichos grupos en función de sus medias retributivas como un indicador de la estructuración del mercado de trabajo.

\section{¿ESTRUCTURA LINEAL O ESTRUCTURA SEGMENTADA?}

En la fase anterior queda demostrada la existencia de diferencias sig. nificativas entre grupos de ingenieros industriales determinados por las diversas modalidades de las vatiables que califican al individuo y al puesto de trabajo. Se ha comprobado asimistno que, por sí solas, las variables que 
tradicionalmente se habían utilizado para calificar a la empresa y configurar situaciones diversas de mercado no tienen ese potencial discriminatorio. Razonamos asimismo el hecho de mantenerlas en los análisis postetiores, en los que intervienen de forma simultánea con todas las otras variables para tratar de encontrar su grado de incidencia en la estructuración del mercado de trabajo.

Teniendo pues en cuenta todo esto, y de acuerdo con nuestro diseño experimental, se trata en este segundo momento de hallar una estructura lineal u ordenación de los ingenieros industriales, construida sobre la base del conjunto de las variables ya conocidas, que resulte consistente con la ordenación salarial empíricamente observada. En definitiva se trata de ver si existe una lógica de ordenación para todos los ingenieros, un metcado de trabajo único para todos, o si por el contrario, comprobando la inexistencia de esta lógica única, se ha de aceptar la hipótesis alternativa de una multiplicidad de lógicas y por lo tanto de segmentos significativamente diferentes del mercado de trabajo de los ingenieros industriales.

El proceso de análisis se articula en cuatro fases diferenciadas: 1) detección de los factores estructurantes; 2) clasificación de los individuos y descripción de las clases; 3) validación de la clasificación obtenida; y 4) contrastación con la ordenación salarial.

Hemos de insistir aquí en que los salarios únicamente intervienen en la última fase y que la clasificación se efectúa exclusivamente en base a lo que hemos denominado variables estructurales.

Para la detección de los factores estructurantes se procedió a someter a las mattices de datos correspondientes a los sucesivos años en las que se contenía la información sobre las variables de cada individuo, al análisis en componentes principales, que tal y como ya se ha apuntado no exige ningún tipo de hipótesis previa de explicación causal. Esta cuestión tesultaba importante para que los factores resultantes fueran absolutamente independientes de cualquier interpretación previa como garantía de que la clasificación que pudiéramos establecer después a partir de ellos resultara objetiva.

El número de factores se limitó a cuatro a partir de pruebas previas en las que se constató que la varianza explicada por los cuatro factores superaba el $70 \%$ de la varianza total para cada uno de los cinco años analizados. Al mismo tiempo se computaron las coordenadas de cada uno de los individuos sobre estos cuatro factores, que después fueron utilizadas para realizar la clasificación. El resultado de este análisis fue la obtención de los factores ortogonales, es decir no cortelacionados entre sí, formados por la combinación lineal de las variables intervenientes y que mejor explicaban la varianza de los conjuntos de observaciones. 
El factor 1, que explica por sí solo entre el $24,8 \%$ y el $26,0 \%$ de la varianza total de cada año, viene caracterizado de forma estable por las variables «función» y «área de funciones». Este factor no presenta ninguna dificultad de interpretación dada la regularidad de las contribuciones de dichas variables. Así, podemos calificar a este factor como un eje Funcrónpostción que, por otra parte, es el que mejor ordena a los individuos. Se trata obviamente de un factor definido por las variables que caracterizan al puesto de trabajo. Es pues el puesto de trabajo el elemento determinante y principal en la estructuración factorial para los diferentes años.

El factor 2 se articula para los cinco años en torno a las variables caracterizadoras de los individuos, «antigüedad en el puesto» y «experiencia profesional», que mantienen este orden de importancia en todos los años excepto para 1975, en el que apatecen invertidos pero con una diferencia numética muy pequeña. Tampoco este factor tiene dificultad de interpretación y se le puede calificar como un factor de ANTIGÜEDAD-EXPERIENCra. La varianza explicada por él oscila entre un $16,8 \%$ y un $18,3 \%$ de la varianza total.

El factor 3 explica entre un $15,6 \%$ y un $16,2 \%$ de la varianza total y se circunscribe en torno a las variables «tamaño» y «procedencia de capital» con excepción del año 1979, en el que la variable distancia a gerencia» sustituye a la segunda. Esta alternancia supone Ia aparición de una indeterminación en la interpretación del factor, aunque puede ser hecha perfectamente en términos de caracterización de la empresa dada la persistencia de tales variables como determinantes del mismo.

En las contribuciones relativas al factor 4 es donde se observan mayores alternancias, si bien se da la presencia continuada de la variable «sector de actividad» en lugar destacado. Por otra parte hay que tesaltar la presencia de la variable «distancia a gerencia» en tres de los cinco casos, con lo que se produce una mezcla entre variables caracterizadoras de la empresa y del puesto de trabajo. Sin embargo, la variable «distancia a gerencia» tiene sus contribuciones muy repartidas entre factores sin que sea nunca la contribución dominante, lo que le hace tener hasta cierto punto un comportamiento errático dentro del conjunto de la estructura factotial. Esta falta de transparencia en la interpretación no tiene mayor trascendencia dada la relativamente escasa aportacion de este factor a la explicación de la varianza, ya que sus porcentajes de explicación oscilan entre un $11,1 \%$ y un $14 \%$.

En la tabla 2 se puede ver una representación sintética de la estructura factorial resultante para los cinco años que contexpla nuestro estudio. 


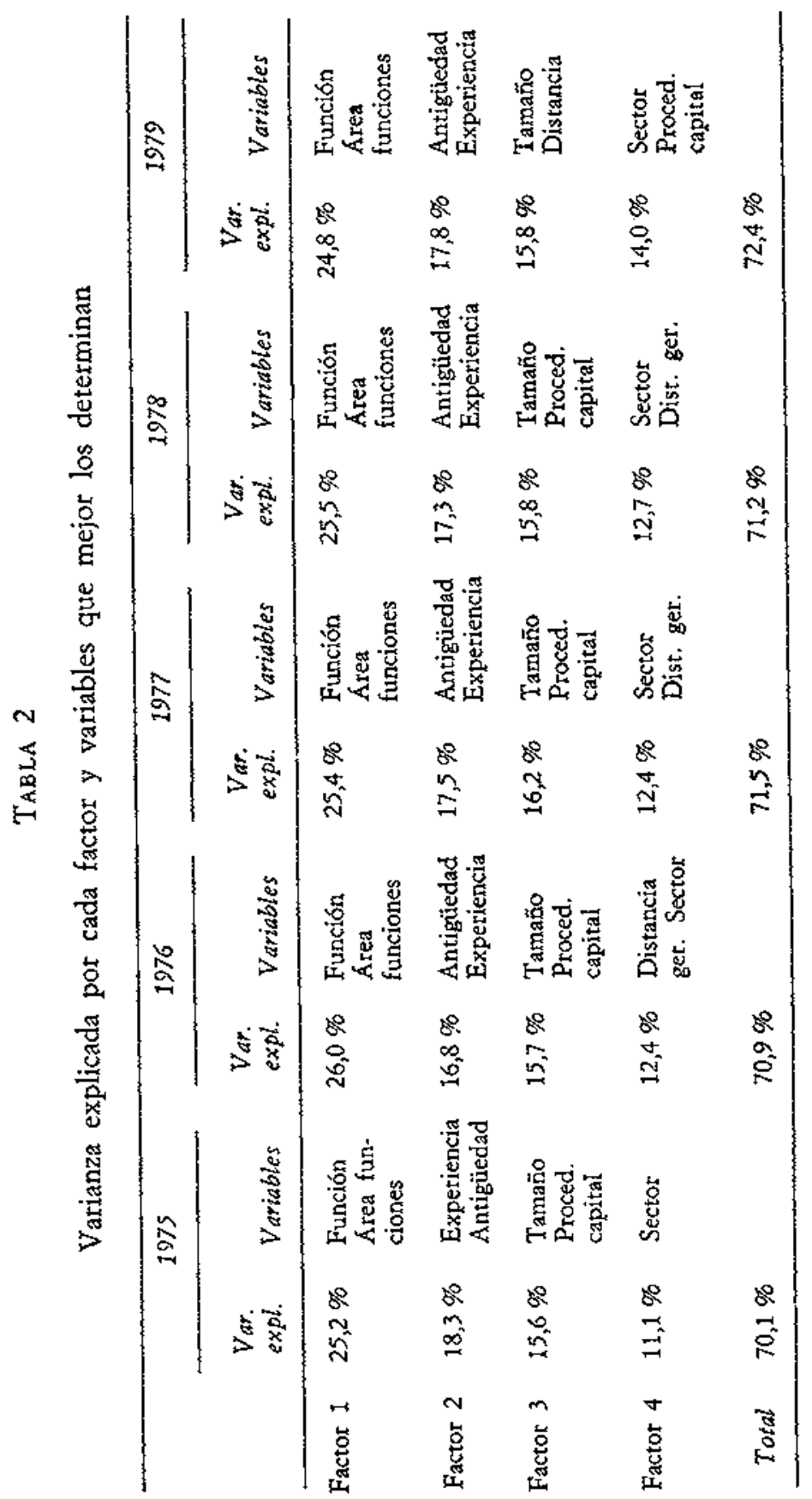


La determinación de estos ejes factoriales tiene como corolario natural el cálculo de las coordenadas de cada uno de los individuos sobre dichos ejes. Ello proporciona una ubicación relativa, en este espacio de dimensión más reducida que el espacio original, de cada uno de los individuos y permite manipular su agrupación por critetios topológicos estrictos sin que interfiera en dicho proceso la diferente interpretación de las variables e incluso la de las modalidades.

Por otra parte hay que destacar que la técnica utilizada tanto para la detección de los factores como para el cálculo de las coordenadas efectúa una operación previa de normalización de los valores empíricamente observados, destinada a evitar los efectos de distorsión producibles por la diferente construcción de las escalas o modalidades.

El procedimiento de clasificación, que ya se explicitó con anterioridad, consiste básicamente en una aplicación concreta de la técnica de las nubes dinámicas, con elección aleatoria de centros y asignación iterativa de los individuos a aquellos grupos de cuyo centro distan menos. En nuestro caso hemos operado con cuatro centros elegidos en cada iteración y una serie de seis iteraciones. Este mecanismo nos permite la obtención de clases estables - nueve- más una que recoge a aquellos individuos que han tenido un comportamiento errático a lo largo de las sucesivas iteraciones.

Un problema no menor era el de los individuos con falta de información en alguna de las variables. Para resolverlo se diseñó el sistema de manera que estos individuos tuvieran una coordenada sobre el factor para el que les faltaba información con valores máximos. De esta manera construíamos ura hiperesfera que contenía a todos aquellos individuos con valores desconocidos en aiguna de las variables y resultabar agrupados durante el proceso de clasificación en una misma clase.

Los resultados concretos de esta clasificación pueden verse en el texto de la tesis y como ayuda a la interpretación de la misma se efectuó un análisis de componentes principales sobre los integrantes de cada una de las clases. Forzosamente la interpretación de esta clasificación tenía que restitar opaca dada la utilización combinada de todas las variables. El aspecto interpretativo resultaría prioritario en caso de que la clasificación obtenida resultara concomitante con la ordenación inducida por los salarios. En la medida que esta concomitancia no se produzca, la interpretación de cada una de las clases puede ser ahorrada. Idénticamente puede ser pospuesta la comparación entre los resultados de la clasificación para los diferentes años. En cualquier caso no tiene demasiado sentido inundat este tesumen de datos numéricos que probablemente diffcultarian el seguimiento argumental de la exposición.

A partir de la clasificación y de su difcultad de interpretación se hacia 
necesario comprobar que no se trataba de una clasificación errática sino que realmente correspondía a la intencionalidad con que se había construido. Esta validación resultaba necesaria para legitimar las conclusiones que pudietan derivarse de su contrastación con la ordenación salarial. Para ello se construyó una nueva clasificación siguiendo una técnica diferente a la utilizada en el ptimer caso, para evitar una comprobación tautológica, y posteriormente contrastar ambas clasificaciones. Si el resultado del contraste era suficientemente significativo en cuanto al paralelismo de las dos ciasificaciones podríamos aceptar la primera como base de nuestros análisis posteriores. Si las clasificaciones resultaban significativamente diferentes habria que desecharla y rectificar el proceso seguido. En esta tesitura había que elegir un procedimiento derivado del análisis discriminante que presentaba la doble ventaja de cumplir los requisitos de diferenciación y mantener el paralelismo con la prueba de contraste de la clasificación con la ordenación salarial.

La pertinencia del análisis discriminante como técnica de confección de grupos resulta probada y su utilización nos daba una lectura «en negativo» de la clasificación al operar por criterios de minimización de la varianza en el interiot de los grupos en vez del critetio de maximización con que opera el análisis factorial.

Las variables discriminantes utilizadas fueron las mismas que se habian utilizado en el factorial y fueron introducidas concurrentemente en el análisis mediante el método directo, que es el más apropiado cuando no se quiere trabajar con agrupaciones jerarquizadas de variables.

La conclusión del contraste de ambas clasificaciones es que se produce un muy alto nivel de concordancia situándose el porcentaje de individuos «bien» clasificados entre el $80 \%$ y el $90 \%$. En algún año se producen ligerísimas desviaciones respecto de estas cotas pero el conjunto de los datos permite dar como buena la clasificación obtenida a partir del análisis factotial de componentes principales.

Ahora, pues, ya estamos en condiciones de realizar la contrastación de esta clasificación con la ordenación que inducen los salarios. La prueba que efectuaremos es estrictamente paralela a la que hemos realizado, con una única variable discriminante que es el salario percibido.

Los porcentajes de individuos «biern» clasificados, es decir aquellos para los que la clasificación y la ordenación salarial son coincidentes, oscilan entre un $14,95 \%$ y un $21,63 \%$. La extraotdinatiamente baja magnitud de estos porcentajes hace pensar en la imposibilidad de que pudieran mejorałse hasta alcanzar cotas significativas de correlación, mediante afinamientos sucesivos. A partir de estos datos podemos afirmar, pues, la inexistencia de una única estructura lineal en la clasificación de los individuos 
según las variables que definen su posición estructutal en el mercado de trabajo, a partir de la constatación de la no correspondencia con la estructura salarial que, efectivamente y como resulta obvio, es lineal.

Ante esta constatación se plantea una alternativa de interpretación: o bien existe una estructuración lineal del mercado de trabajo que resulta de la intervención de toda una serie de variables ciferentes a las utilizadas en nuestro análisis o bien hemos de rechazar la hipótesis de la estructura lineal y aceptar la hipótesis alternativa de una estructuración segmentada, con superposiciones en la banda salarial, y resultante de la coexistencia de diversas lógicas en el interior del mercado de trabajo.

La primera de estas opciones significaría hacer una pirueta teórica en Ia medida en que todas las variables utilizadas siguen siendo vigentes y tomadas en cuenta en los procesos de selección y contratación que se dan en el metcado de trabajo. Incluso aquellas otras variables que no aparecen en nuestra aplicación pero que han sido mencionadas al analizar las relâciones de metcado desde un punto de vista teórico, son o pueden ser entendidas como derivaciones y precisiones de las aquí utilizadas. En este sentido nuestra aplicación habría resultado incompleta debido a la falta de información adicional sobre estas nuevas variables, pero no hubiera podido dar unos resultados tan demostrativos de la ausencia de correlación, ya que en el mejor de los casos hemos hallado correspondencia entre ambas clasificaciones para algo más de la quinta parte de la población, lo cual puede ser entendido en términos perfectamente aleatorios.

Así pues, si la dinámica y la práctica del mercado de trabajo impiden rechazar la pertinencia de las variables utilizadas y éstas se han mostrado tan ineficientes para la construcción de una estructura lineal, lo más correcto desde el punto de vista lógico y metodológico es aceptar la segunda opción de la alternativa diseñada, lo que, además, es perfectamente coherente con nuestros análisis teóricos de apartados prececentes.

La aceptación de la existencia de una estructura segmentada con lógicas propias en el interior del mercado de trabajo supone un cambio radical en la orientación de las investigaciones sobre dicho mercado, y en particular sobre la formación de los salarios. Esta estructuración multisegmentada es incompatible con la vieja idea de las teorías neoclásicas de los salarios como resultantes de una combinación lineal de las variables estructurales definidoras del individuo, el puesto de trabajo o la empresa, en la medida en que to se puede hablar de una función salarial, sino que se habrá de hablar de funciones salariales, tantas como segmentos diferenciados tenga el mercado de trabajo que se analiza. En este sentido la búsqueda de las productividades marginales de la educación o de la experiencia, y, en genetal, las investigaciones derivadas de la teoría de la economía de la educación y del 
capital humano pierden sentido, pues queda tota la hipótesis de homogeneidad del mercado de trabajo.

La alternativa metodológica que aquí se apunta va dirigida, en vez de a dar explicación de la especificidad de situaciones en un contexto homogéneo, a detectar la estructuración de las regularidades observadas en los diversos segmentos heterogéneos del mercado.

\section{INICIANDO LA BUSSQUEDA DE LOS CRITERIOS DE SEGMENT ACION}

Los resultados obtenidos en el análisis anterior demuestran la inexis" tencia de una única lógica de articulación del mercado peto, como todas las demostraciones a contrario, no suministra información acerca de los criterios diversos que presiden la relación entre ubicaciones diferenciadas dentro del sistema productivo y las retribuciones salariales percibidas.

Nuestra intención es iniciar el proceso de búsqueda de estos criterios de segmentación por una vía especulativa que no encuentra su justificación tanto en la rotundidad de los resultados obtenidos como en el desbloqueo conceptual e interpretativo que supone sobre los análisis tradicionales del mercado de trabajo.

Para implementar esta intencionalidad analítica hemos partido de la aceptación, como bipótesis de trabajo, de la teoría de Thurow acerca de la organización del mercado de trabajo en colas y a partir de ella realizamos una lectura recíproca a la efectuada en los anteriores análisis, es decir, desde la remuneración salarial percibida hacia las variables estructurales.

Partimos pues de una compartimentación del colectivo a partir de los salarios percibidos y efectuamos un análisis de los grupos ordenados por rangos de magnitud salarial. Este procedimiento planteaba la dificultad de la construcción de los rangos salariales dado que no disponíamos de información previa para determinar el número de rangos, ni la amplitud de los mismos, así como la posible existencia de una superposición en las zonas extremas de los rangos. Teniendo en cuenta que para nuestro trabajo no interesaban tanto los salarios realmente percibidos como la posición relativa de los individuos en la escala salarial, se optó por construir cinco rangos que agrupasen cada uno de ellos al $20 \%$ correlativo de la pobla. ción estudiada para cada año. De esta manera se trabajaba con una estructuta salarial estable y al tratarse de efectivos numéricamente importantes en cada uno de los cinco rangos se relativizaba el posible sesgo inducido pot las zonas de superposición en los valores extremos de cada rango. 


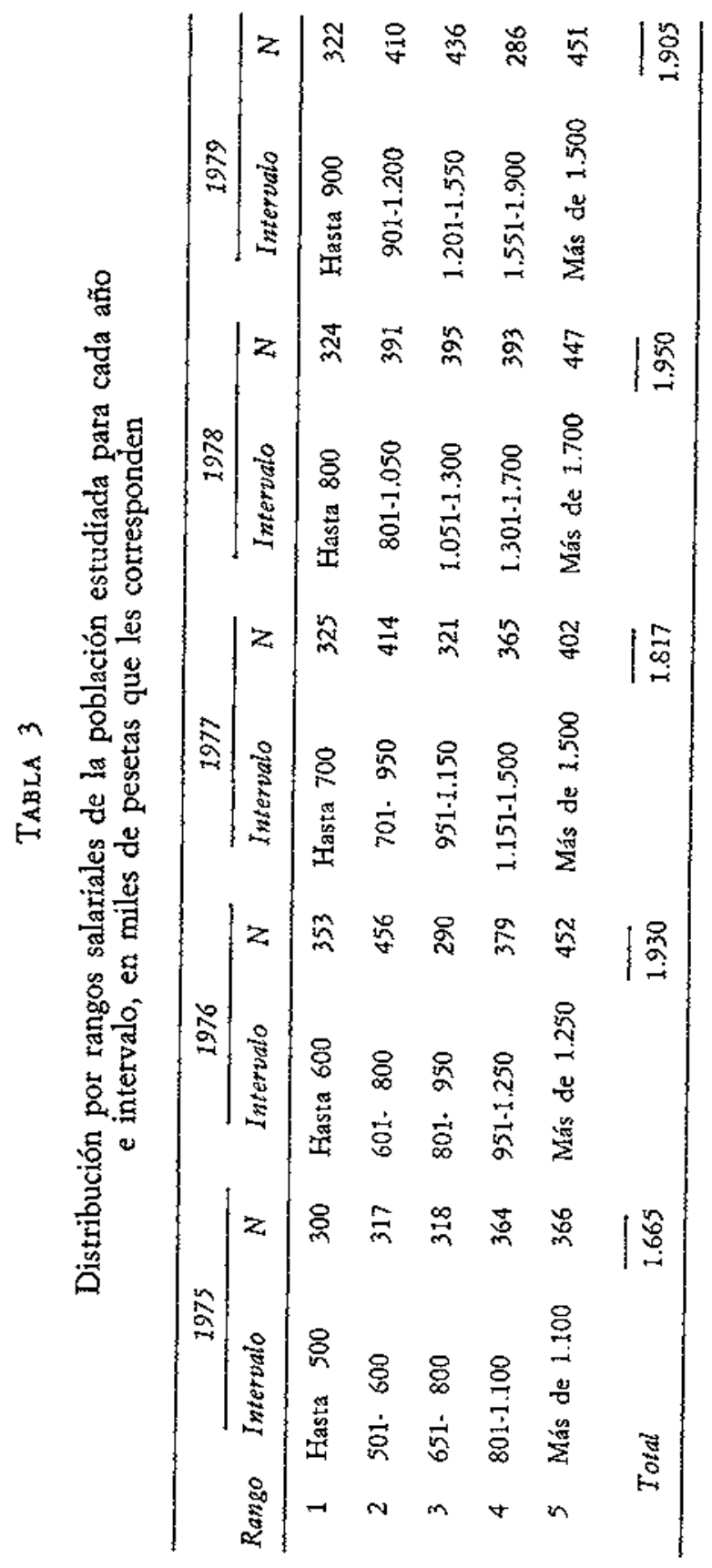


La proporcionalidad de los rangos fue respetada lo más escrupulosamente posible dadas las agtupaciones de individuos en los puntos de la escala salarial. Los límites de los rangos y los efectivos de cada uno de ellos pueden verse en la tabla 3.

Sobre la base de estas distribuciones se tealizó el análisis de cada rango salarial en cuanto a los individuos que lo forman y en cuanto a la detección de los factores estructurales de cada rango a fin y efecto de ver si se obsetvaban diferencias significativas entre ellos que pudieran dar criterios de interpretación al fenómeno de la segmentación.

De los tesultados de este análisis se podía hacer una doble lectura. Por una parte el contraste entre rangos para un mismo año $y$, por otra, la estabilidad o dinámica de las estructuras factoriales de cada uno de los rangos a lo latgo de los diferentes años. Queremos insistir en el carácter exploratorio de nuestro análisis que encuentra su virtus, si es que tiene alguna, en ser una muestra puntual del tipo de análisis a que habrá que someter al mercado de trabajo $\mathrm{y}$, presumiblemente, a otros ámbitos dei conocimiento de las ciencias sociales, para superar el actual impasse analitico e interpretativo en que nos encontramos.

A efectos ilustrativos se adjuntan las tablas tesumen (tablas 4 a 8) de los diversos análisis de componentes principales para cada uno de los rangos construidos. En ellas se puede apreciar la estabilidad de las estructuras factoriales, así como la dinámica de las mismas a lo largo del tiempo. 
La segmentación del mercado de trabajo

\section{TABLA 4}

Resultados del análisis en componentes principales sobre cada uno de los rangos. Año 1975.

Varianza

explicado Variables

\begin{tabular}{|c|c|c|c|}
\hline \multicolumn{4}{|c|}{ Rango 1} \\
\hline Factor & 1 & $22,8 \%$ & Antigüedad $(0,89051)$ Experiencia $(0,86892$ \\
\hline$\gg$ & 2 & $19,3 \%$ & Función $(0,85932)$ Area funcional $(0,81968)$ \\
\hline$»$ & 3 & $14,1 \%$ & Sector $(0,80079)$ Proc. capital $(-0,75284)$ \\
\hline$»$ & 4 & $13,6 \%$ & Distancia $(0,83056)$ Tamaño $(0,70568)$ \\
\hline \multicolumn{2}{|c|}{ Total } & $69,9 \%$ & \\
\hline \multicolumn{4}{|c|}{ Rango 2} \\
\hline Factor & 1 & $21,2 \%$ & Funcion $(0,84014)$ Area funcional $(0,83743)$ \\
\hline$\gg$ & 2 & $19,5 \%$ & Antigüedad $(0,88749)$ Experiencia $(0,88729)$ \\
\hline$\gg$ & 3 & $16,8 \%$ & Tamaño $(0,81732)$ Proc, capital $(0,73945)$ \\
\hline$\gg$ & 4 & $12,7 \%$ & Sector $(0,84466)$ Distancia $(0,46459)$ \\
\hline \multicolumn{2}{|c|}{ Total } & $70,1 \%$ & \\
\hline \multicolumn{4}{|c|}{ Rango 3} \\
\hline Factor & $\mathrm{t}$ & $22,5 \%$ & Área funcional $(0,84324)$ Funcion $(0,84305)$ \\
\hline$\gg$ & 2 & $17,9 \%$ & Antigiedad $(0,86155)$ Experiencia $(0,84487)$ \\
\hline$»$ & 3 & $16,0 \%$ & Proc. capital $(0,65104) /$ Sector $(-0,80304)$ \\
\hline$»$ & 4 & $11,5 \%$ & Distancia $(0,81354)$ Tamaño $(0,47608)$ \\
\hline \multicolumn{2}{|c|}{ Total } & $67,8 \%$ & \\
\hline \multicolumn{4}{|c|}{ Rango 4} \\
\hline Factor & 1 & $24,9 \%$ & Area funcional $(0,88611)$ Función $(0,82790)$ \\
\hline$\gg$ & 2 & $17,8 \%$ & $\begin{array}{l}\text { Proc. capital }(0,75250) \text { Tamaño }(0,53625) \\
\text { / Sector }(-0,74885)\end{array}$ \\
\hline$\triangleright$ & 3 & $16,9 \%$ & Experiencia $(0,84361)$ Antigüedad $(0,83440)$ \\
\hline$\gg$ & 4 & $10,1 \%$ & Distancia $(-0,88004)$ \\
\hline \multicolumn{2}{|c|}{ Total } & $69,7 \%$ & \\
\hline \multicolumn{4}{|c|}{ Rango 5} \\
\hline Factor & 1 & $27,3 \%$ & Area funcional $(0,85171)$ Función $(0,83691)$ \\
\hline$\gg$ & 2 & $16,7 \%$ & Tamaño $(0,81340) /$ Distancia $(-0,67366)$ \\
\hline$»$ & 3 & $13,9 \%$ & Experiencia $(0,82180)$ Antigüedad $(0,76749)$ \\
\hline$»$ & $\gg$ & $11,1 \%$ & Proc. capital $(0,63444) /$ Sector $(-0,81978)$ \\
\hline Tota & & & \\
\hline
\end{tabular}


«Papers»: Revista de Sociologia

\section{TABLA 5}

Resultado del análisis en componentes principales sobre cada uno de los rangos. Año 1976.

\begin{tabular}{|c|c|c|}
\hline & $\begin{array}{l}\text { Varianza } \\
\text { explicada }\end{array}$ & Variables \\
\hline Rango 1 & & \\
\hline Factor 1 & $21,9 \%$ & Antigüedad $(0,87132)$ Experiencia $(0,87087)$ \\
\hline$\gg \quad 2$ & $19,7 \%$ & Area funcional $(0,84720)$ Función $(0,83927)$ \\
\hline$\gg 3$ & $15,2 \%$ & Distancia $(0,81134)$ Tamaño $(0,77291)$ \\
\hline$\gg 4$ & $13,7 \%$ & Sector $(0,79387)$ / Proc. capital $(-0,64385)$ \\
\hline Total & $70,5 \%$ & \\
\hline Rango 2 & & . \\
\hline Factor 1 & $21,3 \%$ & Área funcional $(0,88791)$ Función $(0,88486)$ \\
\hline$» 2$ & $20,0 \%$ & Antigiedad $(0,87494)$ Expexiencia $(0,85653)$ \\
\hline$\gg$ & $16,9 \%$ & Proc. capital $(0,79009) /$ Sector $(-0,79918)$ \\
\hline$\gg 4$ & $14,2 \%$ & Distancia $(0,80690)$ Tamaño $(0,71945)$ \\
\hline Total & $72,4 \%$ & \\
\hline Rango 3 & & \\
\hline Factor 1 & $21,1 \%$ & Función $(0,88838)$ Area funcional $(0,87335)$ \\
\hline$\gg 2$ & $18,5 \%$ & Experiencia $(0,82233)$ Antigüedad $(0,80948)$ \\
\hline$»$ & $15,7 \%$ & $\begin{array}{l}\text { Proc. capital }(0,75651) \text { Tamaño }(0,58495) \\
/ \text { Sector }(-0,58631)\end{array}$ \\
\hline 》 4 & $14,5 \%$ & Distancia $(0,86626)$ \\
\hline Total & $69,9 \%$ & \\
\hline Rango 4 & & \\
\hline Factor 1 & $22,7 \%$ & Area funcional $(0,85728)$ Funcion $(0,84606)$ \\
\hline${ }_{\gg} \quad 2$ & $17,4 \%$ & Antigüedad $(0,83239)$ Experiencia $(0,72842)$ \\
\hline$\gg 3$ & $14,8 \%$ & Sector $(0,77495)$ Distancia $(0,75041)$ \\
\hline$\gg 4$ & $12,5 \%$ & Proc. capital $(0,82722)$ Tamaño $(0,62189)$ \\
\hline Total & $67,5 \%$ & \\
\hline Rango 5 & & \\
\hline Factor 1 & $28,2 \%$ & $\begin{array}{l}\text { Función }(0,86975) \text { Área funcionat }(0,84217) \\
\text { / Distancia }(-0,69429)\end{array}$ \\
\hline$\gg 2$ & $16,2 \%$ & Antigüedad $(0,82163)$ Experiencia $(0,72281)$ \\
\hline$\gg 3$ & $14,4 \%$ & Sector $(0,86010)$ / Tamaño $(-0,53928)$ \\
\hline$\gg 4$ & $11,6 \%$ & Proc. capital $(0,96649)$ \\
\hline Total & $70,4 \%$ & \\
\hline
\end{tabular}


La segmentación del mercado de trabajo

\section{TABLA 6}

Resultados del análisis en componentes principales sobre cada uno de los rangos. Año 1977.

\section{Varianza}

explicada

Variables

$\begin{array}{cccl}\text { Rango } 1 & & \\ \text { Factor } 1 & 25,0 \% & \text { Experiencia }(0,89419) \text { Antigüedad }(0,84554) \\ \Rightarrow & 2 & 20,1 \% & \text { Area funcional }(0,88221) \text { Función }(0,84264) \\ \Rightarrow & 3 & 15,3 \% & \text { Tamaño }(0,78868) \text { Proc. capital }(0,75462) \\ \Rightarrow & 4 & 12,5 \% & \text { Sector }(0,74880) \text { Distancia }(0,70384) \\ & & \end{array}$

\section{Rango 2}

Factor $1 \quad 22,7 \%$

$\gg 218,9 \%$

Antigüedad (0,86517) Experiencia (0,85860)

\# $3 \quad 17,0 \%$

$4 \quad 12,9 \%$

Función $(0,85529)$ Átea funcional $(0,84911)$

Distancia $(0,86588)$ Tamaño $(0,75732)$

Total $\overline{71,5 \%}$

Proc. capital $(0,71831)$ / Sector $(-0,82209)$

\begin{tabular}{ccc} 
Rango 3 & \\
Factor & 1 & $21,3 \%$ \\
$\gg$ & 2 & $19,9 \%$ \\
$\Rightarrow$ & 3 & $15,1 \%$ \\
$\gg$ & 4 & $13,9 \%$ \\
\cline { 2 - 2 } Total & $70,1 \%$
\end{tabular}

$\begin{array}{crr}\text { Rango } & 4 & \\ \text { Factor } & 1 & 26,3 \% \\ \Rightarrow & 2 & 18,0 \% \\ 》 & 3 & 14,4 \% \\ \Rightarrow & 4 & 12,1 \% \\ \text { Total } & \frac{70,8 \%}{}\end{array}$

Area funcional $(0,88860)$ Función $(0,87473)$

Experiencia $(0,85095)$ Antigüedad $(0,84779)$

Dístancia $(0,82482)$ Tamaño $(0,65101)$

Proc. capital $(0,83340) /$ Sector $(-0,50562)$

\section{Rango 5}

Factor $1 \quad 29,1 \%$

\begin{tabular}{lrr}
$\gg$ & 2 & $16,8 \%$ \\
$\gg$ & 3 & $14,4 \%$ \\
& 4 & $11,3 \%$ \\
\cline { 2 - 2 } Total & & $71,6 \%$
\end{tabular}

Función $(0,87101)$ Area funcional $(0,84415)$

Antigüedad $(0,84068)$ Experiencia $(0,80610)$

Pro. capital $(0,81886)$ Tamaño $(0,57527)$

Sector $(0,77455)$ Distancia $(0,69842)$

Función $(0,84946)$ Área
/ Distancia $(-0,71185)$

Antiguiedad $(0,81999)$ Experiencia $(0,79440)$

Proc. capital $(0,90990)$

Sector $(0,98005)$ 
«Papers»: Revista de Sociologia

\section{TABLA 7}

Resultados del análisis en componentes principales sobre cada uno de los rangos. Año 1978.

\begin{tabular}{|c|c|c|}
\hline & $\begin{array}{l}\text { Varianxa } \\
\text { explicada }\end{array}$ & Variables \\
\hline \multicolumn{3}{|l|}{ Rango 1} \\
\hline Factor 1 & $24,9 \%$ & Función $(0,89739)$ Area funcional $(0,89514)$ \\
\hline$\gg 2$ & $21,8 \%$ & Experiencia $(0,90588)$ Antigiedad $(0,90396)$ \\
\hline$\Rightarrow 3$ & $16,0 \%$ & Proc. capital $(0,68483) /$ Sector $(-0,73958)$ \\
\hline$\gg 4$ & $12,0 \%$ & Distancia $(0,90858)$ Tamaño $(0,60535)$ \\
\hline Total & $74,7 \%$ & \\
\hline \multicolumn{3}{|l|}{ Rango 2} \\
\hline Factor 1 & $23,5 \%$ & Antigüedad $(0,88319)$ Experiencia $(0,86751)$ \\
\hline$\gg 2$ & $20,7 \%$ & Funcion $(0,85181)$ Area funcional $(0,84983)$ \\
\hline$\gg 3$ & $15,4 \%$ & Distancia $(0,81261)$ Tamaño $(0,77136)$ \\
\hline$\gg 4$ & $13,5 \%$ & Sector $(0,81376)$ / Proc. capital $(-0,70063)$ \\
\hline Total & $73,1 \%$ & \\
\hline \multicolumn{3}{|l|}{ Rango 3} \\
\hline Factor 1 & $19,8 \%$ & Átea funcional $(0,87298)$ Funcion $(0,85229)$ \\
\hline$\gg 2$ & $19,3 \%$ & Antigüedad $(0,86443)$ Experiencia $(0,83181)$ \\
\hline$\gg 3$ & $17,1 \%$ & Distancia $(0,79060)$ Tamaño $(0,76863)$ \\
\hline$\gg 4$ & $13,4 \%$ & Ptoc. capital $(0,74014) /$ Sector $(-0,72862)$ \\
\hline Total & $69,6 \%$ & \\
\hline \multicolumn{3}{|l|}{ Rango 4} \\
\hline Factor 1 & $23,1 \%$ & Función $(0,85830)$ Area funcional $(0,83350)$ \\
\hline$\gg 2$ & $16,8 \%$ & Antigüedad $(0,81703)$ Experiencia $(0,80124)$ \\
\hline$\gg$ & $14,7 \%$ & Proc. capital $(0,78804)$ Tamaño $(0,62455)$ \\
\hline$»$ & $12,7 \%$ & Sector $(0,79067)$ Distancia $(0,66093)$ \\
\hline Total & $67,4 \%$ & \\
\hline \multicolumn{3}{|l|}{ Rango 5} \\
\hline Factor 1 & $29,1 \%$ & $\begin{array}{l}\text { Función }(0,87338) \text { Átea funcional }(0,82184) \\
\text { / Distancia }(-0,74611)\end{array}$ \\
\hline ». 2 & $17,9 \%$ & Antiguedad $(0,81034)$ Experiencia $(0,79530)$ \\
\hline$\Rightarrow 3$ & $13,9 \%$ & Proc, capital $(0,92942)$ \\
\hline$\$ 4$ & $10,6 \%$ & Sector $(0,96653)$ \\
\hline Total & $71,5 \%$ & \\
\hline
\end{tabular}




\section{TABla 8}

Resultados del análisis en componentes principales sobre cada uno de los rangos. Año 1979.

Varianza

explicada Variables

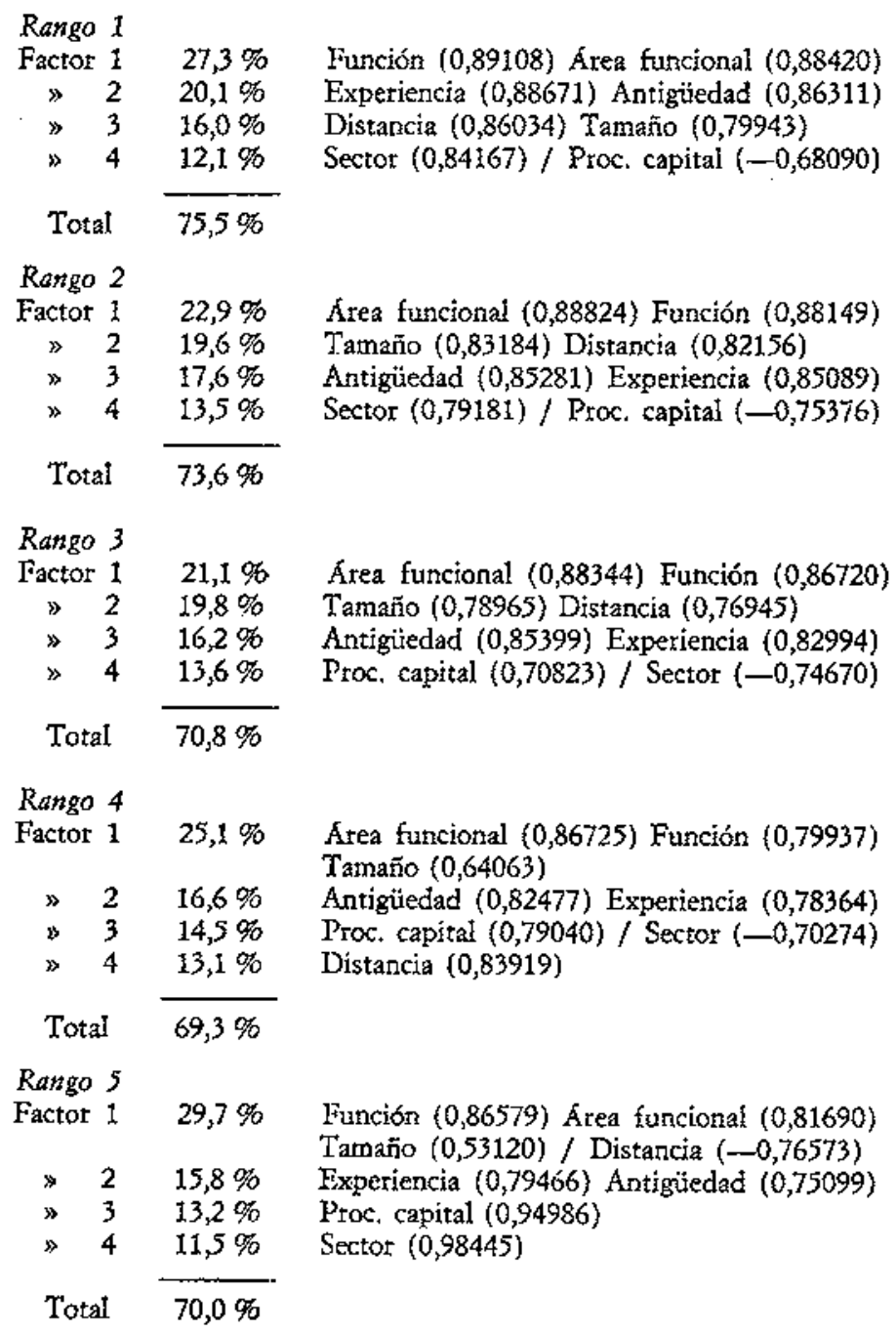

Rango 4

Factor $1 \quad 25,1 \%$

\begin{tabular}{|c|c|}
\hline$\gg \quad 2$ & $16,6 \%$ \\
\hline 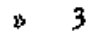 & $14,5 \%$ \\
\hline 4 & $13,1 \%$ \\
\hline Total & $69,3 \%$ \\
\hline
\end{tabular}

Area funcional (0,86725) Función $(0,79937)$ Tamaño $(0,64063)$

Antigüiedad $(0,82477)$ Experiencia $(0,78364)$

Proc. capital $(0,79040) /$ Sector $(-0,70274)$

Distancia $(0,83919)$

Rango 5

Factor $1 \quad 29,7 \%$

Función $(0,86579)$ Área funcional $(0,81690)$

Tamaño $(0,53120)$ / Distancia (-0,76573)

* $215,8 \%$

$3 \quad 3 \quad 13,2 \%$

$\gg 4 \quad 11,5 \%$

Fixperiencia $(0,79466)$ Antigüedad $(0,75099)$

Total $70,0 \%$

Sector $(0,98445)$ 
De los resultados de las mismas cabe concluir que la segmentación del mercado de trabajo es un hecho cierto y que los critetios por los que se rige esta segmentación no son estáticos sino que por el contrario son sensibles a la variación de otras magnitudes, en particular las econónicas, que se relacionan con la creación de oportunidades de empleo. En este sentido hemos de convenir que la segmentación no es explicable desde un modelo estrictamente endógeno.

Se ptreden establecer, no obstante, criterios dominantes en cada momento e incluso se pueden apreciar las características de su transformación en el tiempo. En particular, queda clara la dinámica que ha afectado a los rangos salatiales más bajos con la emergencia de un segmento marginal en el extremo inferior de la banda salarial para el último año analizado y el progresivo desplazamiento de la lógica de un mercado de trabajo estable hacia los rangos salariales superiores. Por otra parte se constata también la altetación de los factores preponderantes en los segmentos de profesionalización en los que el factor de caracterización de la función posición del individuo va desplazando a las características personales de antigüedad y experiencia,

En el tramo superior de la escala salarial se configura un segmento específico de profesionales con mucha experiencia y antigüedad y que ocupan los puestos directivos y altamente ejecutivos. Peto al margen de la configuración específica de los segmentos en cada uno de los momentos analizados, lo que es más importante a retener es la relativamente alta estanqueidad de estos segmentos.

El desarrollo de la carrera profesional de un individuo se realiza en el interior de su propio segmento, puesto que no hay ningún elemento de continuidad entre un segmento y otro. Las variables experiencia y antiguiedad no son suficientes para garantizar el desplazamiento por la escala salarial. Si bien es cierto que todos los individuos que se hallan en el rango superior de la escala tienen niveles altos en ambas, también es cierto, y cada vez más claramente, que no todos los que tienen estos niveles se ubican en el rango superior. El desplazamiento de un rango a otro se produce por «saltos» y éstos quedan reservados para aquellos profesionales que, de acuerdo con su posicionamiento en la estructura y, sobre todo, con su proceso de incorporación al mercado de trabajo, pueden hacerlos.

Por otra parte, en la medida en que la caracterización del puesto de trabajo va siendo más importante en la estructuración de los segmentos y que esta caracterización supone una discontinuidad esencial, la posibilidad de desplazamientos por la vía del cambio de área de funciones y funciones realmente desempeñadas, queda muy limitada.

Como conclusión de todo ello podemos decir que la segmentación del 
mercado de trabajo observada responde a unos criterios prefigurados en el momento de incorporación de los profesionales a dicho mercado, a través de las características de Jas opottunidades de empleo existentes en aquel momento y no tanto de las existentes en el momento en que se analiza, si bien estas últimas pueden tener importancia en el posicionamiento respectivo de los segmentos en la escala salarial.

\section{CONCLUSIONES}

Somos perfectamente conscientes del desajuste que puede existir entre un epígtafe como "Conclusiones», que sugiere una idea de rotundidad y producto acabado, y las elaboraciones de tipo teórico y práctico que se pueden derivar del presente trabajo, en el que hay una vocación explícita* mente orientada a conseguir el desbloqueo del tratamiento teórico y empírico a que convencionalmente se ha sometido al metcado de trabajo.

Una cierta tradición académica, que nos atrevemos a calificar de cientifista y estrecha, ha llevado a establecer como verdaderas conclusiones aquellas que adoptan un aire definitivo y que, de alguna manera, resultan incontestables dado el estado de los conocimientos sobre la cuestión objeto de análisis. Se ha ido produciendo así un cierto olvido de la categoría de provisionalidad en el desarrollo de los conocimientos, que es donde radica realmente el potencial evolutivo del conocimiento, para caet en la esclavitud de un formalismo, muchas veces preciso y perfecto pero que está condenado a repetir y reproducir.

Las conclusiones de nuestro trabajo responden a esta reivindicación epistemológica de la provisionalidad $y$, sin renunciar en modo alguno a los mínimos de corrección exigidos desde el paradigma, se reclaman más como hipótesis de partida de nuevas investigaciones que como productos terminados. A nuestro juicio, las conclusiones de una investigación han de ser forzosamente insatisfactorias como mejor garantía de que no se ha tomado un atajo con la vana pretensión de llegar antes al final. En el proceso del conocimiento no hay atajos $\mathrm{y}$, dicho honradamente, pensamos que tampoco hay un final.

Esto crea, lógicamente, una molesta sensación de inseguridad en el investigador, para el que no queda ni el consuelo de poder dar como buenas aquellas conclusiones insatisfactorias. Tampoco la insatisfacción es la garantía exclusiva de corrección. Sólo el desarrollo de nuevas investigaciones sobre la base de lo provisionalmente establecido y la fecundidad analítica $e$ interpretativa de las mismas pueden proporcionar unas ciertas garantías 
de que se avanza en el proceso de conocer. Nuestras conclusiones, pues, se enmarcan en este contexto epistemológico y sólo en él encuentran su legitimidad.

La más genérica de ellas, y también la más importante desde el punto de vista de la fecundidad interpretativa, es la constatación de la existencia en el mercado de trabajo de una segmentación que presupone la existencia de lógicas diferenciadas en el interior del mismo.

El estudio de la variabilidad de los salatios, a través del análisis del comportamiento de los mismos en función de las variables convencionalmente aceptadas como caracterizadoras del mercado de trabajo, nos demuestra que efectivamente se dan diferencias significativas entre los gupos de ingenieros industriales definidos por las modalidades de alguna de dichas variables. Notoriamente las que mejor permiten discriminar grupos difetenciados son aquellas que hacen referencia a las características del individuo como trabajador.

Sin embargo, ninguna de estas variables por sí sola puede ser tomada como elemento estructurante del mercado de trabajo puesto que resultan, cada una de ellas, absolutamente insuficientes para explicar la vatiabilidad observada en los salatios. Esta constatación nos permite rechazar como carentes de justificación suficiente, no sólo teótica sino también empírica, los estudios de salarios, y en general del mercado de trabajo, derivados de la teoría del capital humano en los que se pretende correlacionar las diferencias salariales observadas con lins diferencias relativas respecto a una variable, mostradas por Ios individu os de un determinado colectivo.

Por otra parte se hace evidente que los estudios sobre dicho metcado han de tomar en consideración conjuntamente toda la batería de variables que son las que, actuando de forma simultánea aunque con importancia diferente, lo estructuran.

La clasificación de los individuos realizada a partir de esta consideración conjunta de todas las variables nos permite afurmar la no existencia de una correspondencia significativa entre la escala salatial y dicha clasiffcación. Esto no es más que el reflejo de la no existencia de una única lógica dentro del mercado de trabajo que, caso de existir, se formalizaría para una mayoría de individuos en una única función que permitiría explicar el salario en base al conjunto de las variables. De ello se deriva la necesidad de coexistencia en el mercado de diversas funciones de formación de los salarios y, en consecuencia, la existencia de lógicas diversas que configuran los denominados segmentos.

La hipótesis adicional de que dichos segmentos tienen un referente en la escala salarial nos permite comprobar la existencia de lógicas de composición diferenciadas para cada uno de ellos en los que experimentalmente 
hemos dividido al colectivo. A pesar de la limitación que supone el hecho de que las posibles zonas superior e inferior respectivamente de los seg. mentos contiguos no son respetadas al establecer puntos de corte singulares en la escala salarial, queda claramente manifiesto que las estructuras factoriales que explican la variabilidad interna de cada segmento son diferentes.

Por otra parte, resulta manifresto que los ctiterios de configuración de los segmentos no son estáticos, sino que son resultado de la interven ción de variables externas sobre el contexto global del mercado de trabajo. Sólo así puede entenderse la emergencia de nuevos segmentos y el desplazamiento de las lógicas respectivas entre los diversos rangos salariales.

El análisis de los resultados obtenidos nos permite elaborar, más como nuevo constructo hipotético que como conclusión definitiva, la interpretación de que cada segmento se articula en base a las sucesivas condiciones dominantes de acceso al mercado de trabajo. Ello es especialmente claro en los segmentos correspondientes a los tangos salariales más bajos. El endurecimiento del mexcado y la situación generalizada de crisis son ele. mentos imprescindibles para la interpretación de la dinámica y emergencia de segmentos mostradas en los resultados.

Estos segmentos muestran una componente notable de estanqueidad. Parece difícil el desplazamiento de un individuo desde un segmento a otro una vez sobrepasado el nivel mínimo de profesionalización. El aumento de experiencia o de antigücdad no es garantía de salto de un segmento a otro en la medida en que son sobre todo las características del puesto de trabajo las que determinan las peculiaridades de los segmentos. Las ca. rreras profesionales tienden a desarrollarse en el interior de los segmentos, y sólo en aquellos casos en que se produce un cambio importante en la función desempeñada $\mathrm{y} / \mathrm{o}$ en el área de funciones podremos hablar de un salto de un segmento a otro del mercado de trabajo.

En general aparecen claramente diferenciados tres segmentos. Correspondiendo a los rangos salariales inferiores encontramos el primero, que contemplaría al contingente de ingenieros que se han incorporado recientemente al mercado de trabajo y que se hallan en fase de profesionalización. En los rangos salariales superiores encontramos un segmento correspondiente a aquellos ingenieros que han alcanzado puestos ejecutivos y gerenciales y que está formado por ingenieros que se incorporaron al mercado de trabajo hace más tiempo. En los rangos salariales centrales aparece un segmento para el que la lógica fundamental es el desarrollo de la profesionalidad en las funciones de tipo eminentemente técnico.

Aparecen pues claramente ligadas las lógicas estructurantes de cada segmento a las condiciones cambiantes de acceso al mercado de trabajo do- 
minantes para cada grupo en el momento de su incorporación. Ello no quiere decir que la modificación postetior de las condiciones generales del mercado no tenga incidencia sobre la dinámica interna de cada segmento como, por ejemplo, el endurecimiento del mercado derivado de la crisis económica que provoca entre otras cosas la ampliación del segmento correspondiente a la fase de primera profesionalización a prácticamente el $40 \%$ de la población.

Resulta especialmente sugerente la emergencia en el rango salarial inferior de un segmento cuya logica estructural es totalmente análoga a la del correspondiente al de desarrollo de la profesionalidad. Interpretado en patata de crisis y endurecimiento puede hablarse de un segmento constituido por puestos de trabajo creados marginalmente en el período de recesión y que únicamente tienen como característica diferencial la de estar mucho menos retribuidos. Aunque sería precisa más información sobre las condiciones de trabajo de estos puestos para corroborar esta interpretación, no cabe duda de que se trata de un segmento de nuevo tipo que no tiene nada que ver con el de primera profesionalización que característicamente había ocupado los rangos salariales inferiores.

Como puede fácilmente apreciarse, la capacidad de sugerencia de estas conclusiones comporta necesariamente la característica de insatisfacción a la que antes hacíamos referencia y que sólo podrá resolverse, aunque sea parcialmente, con el desartollo de nuevas investigaciones realizadas en base a las elaboraciones teórica y metodológica aquí presentadas y que han mostrado suficientemente su capacidad analítica e interpretativa. 


\section{BIBLIOGRAFIA CITADA}

Arrow, K. J., Capron, W. M. Dinamic Shortages and Price Rises: The Engineers-Scientists case, en "Quarterly Journal of Economics", vol. 73, núm. 2, 1959.

Berg, I. Education and Jobs. The Great Training Robbery, Nueva York, Preager, 1970.

Blaug, M., Layard, P. R. G., Woodhall, M. The causes of graduate unemployment in India, Londres, Allen Lane, The Penguin Press, 1969.

Bowles, S., Gintis, H. Schooling in Capitalist America, Nueva York, Basic Books, 1976.

- The problem with Human Capital Theory. A Marxian Critique, en «The American Economic Review», mayo 1975.

Coriat, B. Différentiation et segmentation de la force de travail dans les industries de processus, París, Galilée, 1978.

Doeringer, P., Piore, M. Internal Labor Market and Manpower Analysys, Massachusetts, Heath Lexington, 1971.

Gabinete de Estudios del C.O.I.I.C. Informe sobre la encuesta de Salarios, años 1976, 1977, 1978, 1979, 1980, Barcelona, Colegio Oficial de Ingenieros Industriales de Cataluña.

Gintis, H. The nature of Labor Exchange and the Theory of Capitalist Production, en «Review of Radical Political Economics», vol. 8, núm. 2, 1976.

Gordon, D. From Steam Whistles to Coffee Breaks, Nueva York, Dissent, 1972.

-, Reich, M., Edwards, R. C. A Theory of Labor Market Segmentation, en «The American Economic Review», mayo 1973.

Lebart, L., Morineau, A., Tabard, W. Tecbniques de la déscription statistique, Paris, Dunod, 1977.

Paci, M. Mercato dei Laboro e Classi Soziali in Italia, Bolonia, il Mulino, 1973.

Piore, M. Fragments of a Sociological Theory of Wages, en «American Economic Association», mayo 1973.

Rawlins, U. L., Ulman, Ll. «The utilisation of College - Trained Man- 
«Papers»: Revista de Sociologia

power in the United States», en Gordon, Margaret, S., ed., Higher Education and the Labor Market, Nueva York, McGraw Hill, 1974. Thurow, L. C. Generating inequality, Nueva York, Basic Books, 1975.

«Measuring the economic benefits of education», en Gordon, Margaret, S., op. cit., 1974.

Vietorisz, T., Hartison, B. Labor Market Segmentation: Positive Feedback and Divergent Development, en «The American Economic Review», mayo 1973. 